\title{
Amplitudes separation and strong-electromagnetic relative phase in the $\psi(2 S)$ decays into baryons
}

\author{
Rinaldo Baldini Ferroli \\ INFN Laboratori Nazionali di Frascati, I-00044 Frascati, Italy \\ Alessio Mangoni॰* \\ INFN Sezione di Perugia, I-06100 Perugia, Italy
}

Simone Pacettiఠ

INFN Sezione di Perugia, I-06100 Perugia, Italy and Università di Perugia, I-06100 Perugia, Italy

Kai Zhu

Institute of High Energy Physics, I-100049 Beijing, People's Republic of China

(Received 30 September 2020; accepted 9 December 2020; published 6 January 2021)

\begin{abstract}
In the framework of a phenomenological model based on an effective Lagrangian, that allows us to exploit all available data, we have obtained the strong, electromagnetic and mixed strong-electromagnetic amplitudes of the $\psi(2 S)$ decays into baryon-antibaryon pairs. The analysis, repeating the procedure successfully implemented in the case of the leading vector charmonium $J / \psi$, revealed a new and quite intriguing phenomenon, that by itself triggered a further quite interesting finding. The phenomenon is the high affinity of the $\psi(2 S)$ meson with the $\Lambda \bar{\Lambda}$ pair, at a level that cannot be justified by the model. As a consequence, we excluded the $\Lambda \bar{\Lambda}$ channel from the set of experimental constraints by largely improving the description power of the model, which then led to another interesting result: the QCD regime turned out to be perturbative already at the $\psi(2 S)$ mass. Finally, the comparison with the results obtained for the $J / \psi$ has shown that the main difference with respect to the $\psi(2 S)$ concerns the $\mathrm{SU}(3)$ breaking source due to the quark mass difference. As expected, consequently to its larger mass, the effects of such a difference are less important for the $\psi(2 S)$ with respect to the $J / \psi$ meson.
\end{abstract}

DOI: 10.1103/PhysRevD.103.016005

\section{INTRODUCTION}

Since their discovery, the $c \bar{c}$ mesons, the so-called charmonia, have been representing unique tools to expand our knowledge on the dynamics of the strong interaction at various energy ranges.

The hadronic decays of the $J / \psi$ meson, a charmonium with quantum numbers $I^{G}\left(J^{P C}\right)=0^{-}\left(1^{--}\right)$, mass $M_{J / \psi} \simeq$ $3.1 \mathrm{GeV}$, and width $\Gamma_{J / \psi} \simeq 9.3 \times 10^{-5} \mathrm{GeV}$ [1], have been deeply investigated. Recently, it has been found that they occur halfway between the perturbative and nonperturbative QCD regime [2]. Moreover, it has been shown that the mixed strong-electromagnetic (strong-EM) amplitude of the $J / \psi$ decays is not always negligible [3].

\footnotetext{
*alessio.mangoni@pg.infn.it
}

Published by the American Physical Society under the terms of the Creative Commons Attribution 4.0 International license. Further distribution of this work must maintain attribution to the author(s) and the published article's title, journal citation, and DOI. Funded by SCOAP.
The procedure to single out the strong, the EM and the mixed strong-EM amplitudes of the decay of a charmonium state into baryon-anti-baryon $(\mathcal{B} \overline{\mathcal{B}})$ pairs belonging to the spin-1/2 SU(3) baryon octet has been defined and implemented for the first time in the case of the $J / \psi$ meson [2].

Such a procedure is based on an effective Lagrangian including $\mathrm{SU}(3)$ symmetry breaking terms, depending on a set of coupling constants to be determined by means of a $\chi^{2}$ minimization.

The whole datasets made available by the Particle Data Group (PDG) [1], together with new results provided by the BESIII Collaboration [4] have been used.

In the case of the $J / \psi$, the strong, the EM and the mixed strong-EM contributions to the total branching ratio (BR), as well as a strong-EM relative phase $\varphi_{J / \psi}=(73 \pm 8)^{\circ}$ have been determined [2].

The $\psi(2 S)$ is a charmonium vector meson usually regarded as the first orbit excited state of $J / \psi$. It has the same quantum numbers, i.e., $I^{G}\left(J^{P C}\right)=0^{-}\left(1^{--}\right)$, mass $M_{\psi(2 S)} \simeq 3.7 \mathrm{GeV}$ and width $\Gamma_{\psi(2 S)} \simeq 2.9 \times 10^{-4} \mathrm{GeV}[1]$. 
It is natural to expect that the decays of both, $J / \psi$ and $\psi(2 S)$, into the same $\mathcal{B} \overline{\mathcal{B}}$ final states do proceed through similar decay mechanisms. Nevertheless, there is experimental evidence that in some channel, as for instance: $e^{+} e^{-} \rightarrow J / \psi \rightarrow \Sigma^{0} \bar{\Sigma}^{0}, \Lambda \bar{\Lambda}$ and $e^{+} e^{-} \rightarrow \psi(2 S) \rightarrow \Sigma^{0} \bar{\Sigma}^{0}$, $\Lambda \bar{\Lambda}$, the decays have significantly different angular distributions $[4,5]$.

It follows that a deeper study of the dynamics of the $\psi(2 S)$ decays into baryon-antibaryon pairs, in the same theoretical framework that has been used for the $J / \psi$ [2], would be quite useful.

The paper is organized as it follows. First, we briefly reviewed the model based on an effective strong Lagrangian that is used to parametrize the decay amplitudes in terms of a set of coupling constants. Second, we explicitly outlined the procedure to build up the amplitudes, by considering the three contributions: purely strong, purely EM and mixed strong-EM. The latter, in particular, is related to the ratio of the mixed-to-strong amplitude $R$, just as for the $J / \psi$ meson. However, it was in this study that the parameter $R$ played the crucial role of revealing that already at the $\psi(2 S)$ mass the QCD does operate in perturbative regime.

Finally, we studied the $\chi^{2}$ function, exploited to determine the coupling constants by fitting to the data on the branching ratios (BRs) of the $\psi(2 S)$ decays and we noticed an anomalous affinity, inexplicable by the model, of the $\psi(2 S)$ itself with the $\Lambda \bar{\Lambda}$ channel. As a consequence, we made the analysis by not using the experimental constraint of the $\Lambda \bar{\Lambda}$ channel.

The parametrization at the amplitude level did allow to extract also the relative phase between strong and EM amplitudes, and the obtained value is compatible with the hypothesis of orthogonality, being $\varphi=(87 \pm 15)^{\circ}$.

\section{THE EFFECTIVE LAGRANGIAN MODEL}

The decays $\psi(2 S) \rightarrow \mathcal{B} \overline{\mathcal{B}}$, where $\mathcal{B}$ stands for a spin- $1 / 2$ baryon belonging to the flavor $\mathrm{SU}(3)$ octet, represented by the matrix

$$
B=\left(\begin{array}{ccc}
\Lambda / \sqrt{6}+\Sigma^{0} / \sqrt{2} & \Sigma^{+} & p \\
\Sigma^{-} & \Lambda / \sqrt{6}-\Sigma^{0} / \sqrt{2} & n \\
\Xi^{-} & \Xi^{0} & -2 \Lambda / \sqrt{6}
\end{array}\right),
$$

can be studied by means of the procedure developed and successfully implemented in the case of the $J / \psi$ meson.

The effective Lagrangian density $\mathcal{L}$, which describes the coupling of the $\psi(2 S)$ to baryon-antibaryon pairs $\mathcal{B} \overline{\mathcal{B}}$, and hence its decays $\psi(2 S) \rightarrow \mathcal{B} \overline{\mathcal{B}}$, is formally defined as [2]

$$
\mathcal{L}=C \operatorname{Tr}(B \bar{B})+[\text { symmetry-breaking terms }],
$$

where $C$ is a constant and the SU(3) symmetry-breaking terms are due to EM and quark-mass-difference effects.
The EM breaking effects, depending on the quark electric charges, i.e., from their coupling with the photon, are given by the four-current

$$
\bar{q} \gamma^{\mu} \Lambda_{\mathrm{E}} q \equiv \frac{2}{3} \bar{u} \gamma^{\mu} u-\frac{1}{3} \bar{d} \gamma^{\mu} d-\frac{1}{3} \bar{s} \gamma^{\mu} s,
$$

where the matrix $\Lambda_{E}$ can be expressed as the combination of the third, $\lambda_{3}$, and the eighth, $\lambda_{8}$, Gell-Mann matrix, i.e.,

$$
\Lambda_{\mathrm{E}}=\frac{1}{2}\left(\lambda_{3}+\frac{\lambda_{8}}{\sqrt{3}}\right)
$$

Similarly, the quark-mass-difference breaking effects are related to the mass term

$$
\bar{q} \Lambda_{\mathrm{M}} q \equiv m_{u} \bar{u} u+m_{d} \bar{d} d+m_{s} \bar{s} s,
$$

and the corresponding matrix $\Lambda_{M}$, in terms of the GellMann matrices and the identity $I$, has the form

$$
\Lambda_{\mathrm{M}}=m_{0} I+\frac{m_{d}-m_{s}}{\sqrt{3}} \lambda_{8}+\frac{m_{u}-m_{d}}{6}\left(2 I+3 \lambda_{3}+\sqrt{3} \lambda_{8}\right),
$$

where

$$
m_{0}=\frac{m_{u}+m_{d}+m_{s}}{3} .
$$

We keep the SU(2) symmetry exact, assuming that $m_{u}=m_{d}$, and hence isospin conservation, so that

$$
\Lambda_{\mathrm{M}}=m_{0} I+\frac{m_{d}-m_{s}}{\sqrt{3}} \lambda_{8}, \quad m_{0}=\frac{2 m_{d}-m_{s}}{3} .
$$

Therefore, the SU(3) symmetry breaking terms are related to the so-called spurion matrices

$$
S_{e}=g_{e} \Lambda_{\mathrm{E}}, \quad S_{m}=g_{m}\left(\Lambda_{\mathrm{M}}-m_{0} I\right),
$$

where $g_{e}$ and $g_{m}$ are the coupling constants. The full Lagrangian density is obtained by adding to the leading term proportional to $\operatorname{Tr}(B \bar{B})$, further terms proportional to [6]

$$
\operatorname{Tr}(\{B, \bar{B}\} S), \quad \operatorname{Tr}([B, \bar{B}] S),
$$

with $S \in\left\{S_{e}, S_{m}\right\}$. The complete Lagrangian density is then defined as

$$
\begin{aligned}
\mathcal{L}= & g \operatorname{Tr}(B \bar{B})+d \operatorname{Tr}\left(\{B, \bar{B}\} S_{e}\right)+f \operatorname{Tr}\left([B, \bar{B}] S_{e}\right) \\
& +d^{\prime} \operatorname{Tr}\left(\{B, \bar{B}\} S_{m}\right)+f^{\prime} \operatorname{Tr}\left([B, \bar{B}] S_{m}\right),
\end{aligned}
$$

where $g, d, f, d^{\prime}, f^{\prime}$ are coupling constants. 
Such a Lagrangian density allows us to parametrize the subamplitudes for the decays $\psi(2 S) \rightarrow \mathcal{B} \overline{\mathcal{B}}$. For more details please refer to Ref. [2].

\section{THE AMPLITUDE SEPARATION}

The amplitude of the generic decay $\psi(2 S) \rightarrow \mathcal{B} \overline{\mathcal{B}}$ can be decomposed as the sum of three contributions

$$
\mathcal{A}_{\mathcal{B} \overline{\mathcal{B}}}=\mathcal{A}_{\mathcal{B} \overline{\mathcal{B}}}^{g g g}+\mathcal{A}_{\mathcal{B} \overline{\mathcal{B}}}^{\gamma}+\mathcal{A}_{\mathcal{B} \overline{\mathcal{B}}}^{g g \gamma}
$$

The sub-amplitudes $\mathcal{A}_{\mathcal{B} \overline{\mathcal{B}}}^{g g g}, \mathcal{A}_{\mathcal{B} \overline{\mathcal{B}}}^{\gamma}$ and $\mathcal{A}_{\mathcal{B} \overline{\mathcal{B}}}^{g g \gamma}$ are related to the three dominant decay mechanisms: purely strong, i.e., mediated by three gluons; purely EM, through one-photon exchange; mixed strong-EM, via two gluons and one photon. The Feynman diagrams of these intermediate processes are shown in Fig. 1.

By taking advantage of the Lagrangian density of Eq. (1), the amplitudes of each $\mathcal{B} \overline{\mathcal{B}}$ final state can be

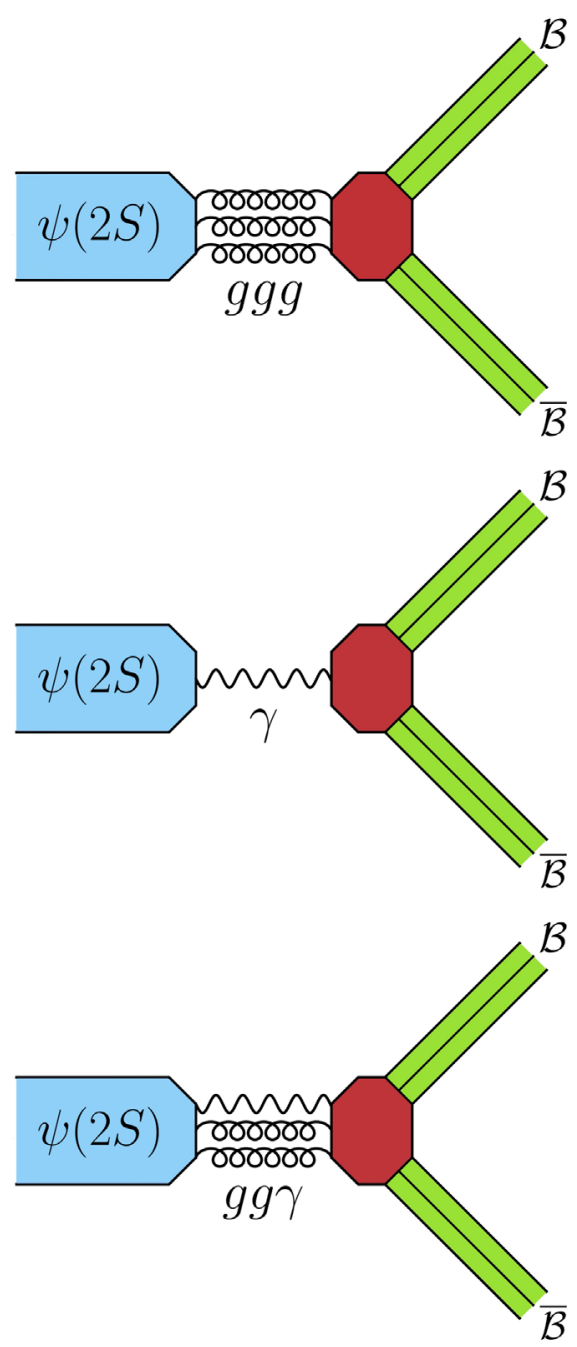

FIG. 1. Feynman diagrams of the strong, EM, and mixed strong-EM contributions for the decay $\psi(2 S) \rightarrow \mathcal{B} \overline{\mathcal{B}}$.
TABLE I. Parametrizations of the $\mathcal{B \mathcal { B }}$ decay amplitudes.

\begin{tabular}{lccc}
\hline \hline $\mathcal{B} \overline{\mathcal{B}}$ & $\mathcal{A}_{\mathcal{B} \overline{\mathcal{B}}}^{g g g}$ & $\mathcal{A}_{\mathcal{B} \overline{\mathcal{B}}}^{g g \gamma}$ & $\mathcal{A}_{\mathcal{B} \overline{\mathcal{B}}}^{\gamma}$ \\
\hline$p \bar{p}$ & $\left(G_{0}-D_{m}+F_{m}\right) e^{i \varphi}$ & $\mathcal{A}_{p \bar{p}}^{g g g} R$ & $D_{e}+F_{e}$ \\
$n \bar{n}$ & $\left(G_{0}-D_{m}+F_{m}\right) e^{i \varphi}$ & 0 & $-2 D_{e}$ \\
$\Lambda \bar{\Sigma}^{0}+$ c.c. & 0 & 0 & $\sqrt{3} D_{e}$ \\
$\Lambda \bar{\Lambda}$ & $\left(G_{0}-2 D_{m}\right) e^{i \varphi}$ & 0 & $-D_{e}$ \\
$\Sigma^{+} \bar{\Sigma}^{-}$ & $\left(G_{0}+2 D_{m}\right) e^{i \varphi}$ & $\mathcal{A}_{\Sigma^{+} \bar{\Sigma}^{-}} R$ & $D_{e}+F_{e}$ \\
$\Sigma^{-} \bar{\Sigma}^{+}$ & $\left(G_{0}+2 D_{m}\right) e^{i \varphi}$ & $\mathcal{A}_{\Sigma^{-} \bar{\Sigma}^{+}} R$ & $D_{e}-F_{e}$ \\
$\Sigma^{0} \bar{\Sigma}^{0}$ & $\left(G_{0}+2 D_{m}\right) e^{i \varphi}$ & 0 & $D_{e}$ \\
$\Xi^{0} \bar{\Xi}^{0}$ & $\left(G_{0}-D_{m}-F_{m}\right) e^{i \varphi}$ & 0 & $-2 D_{e}$ \\
$\Xi^{-} \bar{\Xi}^{+}$ & $\left(G_{0}-D_{m}-F_{m}\right) e^{i \varphi}$ & $\mathcal{A}_{\Xi^{-} \bar{\Xi}^{+}}^{g g g}$ & $D_{e}-F_{e}$ \\
\hline \hline
\end{tabular}

parametrized as shown in Table I [2], where the quantities $G_{0}, D_{m}, F_{m}, D_{e}$, and $F_{e}$ are combinations of the original coupling constants $g, d, f, d^{\prime}, f^{\prime}$, and hence are themselves coupling constants. In particular, $G_{0}$ is related to the $\mathrm{SU}(3)$ exact symmetry; $D_{m}$ and $F_{m}$ account for the quark-massdifference breaking term; $D_{e}$ and $F_{e}$ for the EM one; $\varphi$ is the relative phase between the strong and the EM amplitudes.

The mixed strong-EM amplitude is null for the decays into neutral particles $[2,7,8]$.

By considering an infinite series of excited vector charmonia, with increasing masses $M_{\psi}$, so that the mixed strong-EM and strong amplitudes become functions of $M_{\psi}^{2}$, asymptotically, i.e., as $M_{\psi}^{2}$ diverges, the ratio

$$
R\left(M_{\psi}^{2}\right)=\frac{\mathcal{A}_{\mathcal{B} \overline{\mathcal{B}}}^{g g \gamma}\left(M_{\psi}^{2}\right)}{\mathcal{A}_{\mathcal{B} \overline{\mathcal{B}}}^{g g g}\left(M_{\psi}^{2}\right)}
$$

would tend to a real limit. The high- $q^{2}$ trend of this ratio can be also inferred by the perturbative QCD (pQCD) [9], that provides the asymptotic behavior

$$
R_{\mathrm{pQCD}}\left(q^{2}\right) \underset{q^{2} \gg \Lambda_{\mathrm{QCD}}^{2}}{\sim}-\frac{4}{5} Q_{\mathcal{B}} \frac{\alpha}{\alpha_{S}\left(q^{2}\right)},
$$

where $Q_{\mathcal{B}}$ is the baryon electric charge in units of the positron charge, $\alpha$ and $\alpha_{S}\left(q^{2}\right)$ are the fine structure constant and the QCD running coupling constant, while $\Lambda_{\mathrm{QCD}}$ is the QCD scale and it is of the order of a few hundreds of $\mathrm{MeV}$. In the case of the $J / \psi$ meson, it has been found [2] that the scenario phenomenologically preferred by the whole available dataset on the decays $J / \psi \rightarrow \mathcal{B} \overline{\mathcal{B}}$ was that of having a single value of $R$, not depending on the baryon electric charge. Such a result can be interpreted as the effect of a decay mechanism dominated by still non-pQCD interactions.

As a consequence, we have considered a unique value of the ratio $R$, as the starting hypothesis also in the case of the $\psi(2 S)$ meson. In other words, we started our analysis by assuming that the QCD regime at the $\psi(2 S)$ mass did 
remain nonperturbative, despite the fact that the $\psi(2 S)$ mass is larger than the that of the $J / \psi$.

In the same line of reasoning, in our model, a common relative phase $\varphi$ between strong and EM amplitudes has been used for all the baryons of the flavor SU(3) octet. Moreover, the strong and the mixed amplitudes are assumed to be relatively real, this implies the reality condition for the ratio $R$.

\section{THE ELECTROMAGNETIC COUPLINGS AND THE $\chi^{2}$ DEFINITION}

Since the cross section of the annihilation $e^{+} e^{-} \rightarrow p \bar{p}$ has been measured with high accuracy by the $B A B A R$ experiment [10] and recently by BESIII [11], its value at the mass of the $\psi(2 S)$ meson can be exploited as a further constraint besides the BRs of its decays into baryon pairs. This particular cross section value has been obtained by fitting the BABAR and BESIII data and then by evaluating the fit function at the desired energy, namely $q^{2}=M_{\psi(2 S)}^{2}$, where $q$ is four-momentum of the $e^{+} e^{-}$system in its own center of mass frame. We used the fit function defined in Ref. [11], i.e.,

$$
\sigma_{\mathrm{fit}}\left(q^{2}\right)=\frac{A_{p}\left(1+2 M_{p}^{2} / q^{2}\right)}{\left(q^{2}\right)^{5}\left(\ln ^{2}\left(q^{2} / \Lambda_{\mathrm{QCD}}^{2}\right)+\pi^{2}\right)^{2}},
$$

where $M_{p}$ is the proton mass. The analytic form of this function has been obtained by considering for the electromagnetic form factors the power-law behavior predicted by the pQCD $[12,13]$. It depends on the unique dimensional free parameter $A_{p}$, to be determined by a standard $\chi^{2}$ minimization procedure, and includes the logarithmic QCD correction with $\Lambda_{\mathrm{QCD}}=0.35 \mathrm{GeV}$. Moreover, to avoid the threshold energy regions, where the function of Eq. (3) could fail in describing the cross section, only data at $q^{2}>(2.8 \mathrm{GeV})^{2}$ have been considered in the minimization procedure. Using the cross section value at the $\psi(2 S)$ mass, the EM BR for the decay $\psi(2 S) \rightarrow p \bar{p}$ is obtained as [3]

$$
\begin{aligned}
\mathrm{BR}_{p \bar{p}}^{\gamma} & =\mathrm{BR}_{\mu \mu} \frac{\sigma_{e^{+} e^{-} \rightarrow p \bar{p}}\left(M_{\psi(2 S)}^{2}\right)}{\sigma_{e^{+} e^{-} \rightarrow \mu^{+} \mu^{-}}\left(M_{\psi(2 S)}^{2}\right)} \\
& =(1.35 \pm 0.14) \times 10^{-6}
\end{aligned}
$$

where $\mathrm{BR}_{\mu \mu}=(8.0 \pm 0.8) \times 10^{-6}$ [1] is the $\mathrm{BR}$ of the decay $\psi(2 S) \rightarrow \mu^{+} \mu^{-}$, mediated by one-photon exchange, and $\sigma_{e^{+} e^{-} \rightarrow \mu^{+} \mu^{-}}^{0}\left(q^{2}\right)$ represents the bare $e^{+} e^{-} \rightarrow \mu^{+} \mu^{-}$cross section

$$
\sigma_{e^{+} e^{-} \rightarrow \mu^{+} \mu^{-}}^{0}\left(q^{2}\right)=\frac{4 \pi \alpha^{2}}{3 q^{2}}
$$

A further constraint is given by the well known BR for the decay $\psi(2 S) \rightarrow \Lambda \bar{\Sigma}^{0}+$ c.c., that, being purely EM under the hypothesis of isospin conservation, determines univocally the value of the EM coupling constant

$$
D_{e}=(1.25 \pm 0.07) \times 10^{-4} \mathrm{GeV} \text {, }
$$

as has been discussed in Ref. [14].

The $\chi^{2}$ function has been defined by including all the measured BRs, as well as the constraint from the $e^{+} e^{-} \rightarrow$ $p \bar{p}$ cross section, given in Eq. (4) and represented by the symbol $\mathcal{B} \overline{\mathcal{B}}=p \bar{p}_{\gamma}$,

$$
\chi^{2}(\xi)=\sum_{\mathcal{B} \overline{\mathcal{B}} \in \Omega}\left(\frac{\mathrm{BR}_{\mathcal{B} \overline{\mathcal{B}}}^{\text {th }}-\mathrm{BR}_{\mathcal{B} \overline{\mathcal{B}}}^{\text {exp }}}{\delta \mathrm{BR}_{\mathcal{B} \overline{\mathcal{B}}}^{\exp }}\right)^{2},
$$

where $\xi$ is the set of the six free parameters

$$
\xi=\left\{G_{0}, D_{m}, F_{e}, F_{m}, R, \varphi\right\},
$$

that are the coupling constants of the effective Lagrangian density given in Eq. (1), and the sum runs over the seven baryon pairs of the set

$\Omega=\left\{p \bar{p}_{\gamma}, \Sigma^{0} \bar{\Sigma}^{0}, \Lambda \bar{\Lambda}, p \bar{p}, n \bar{n}, \Sigma^{+} \bar{\Sigma}^{-}, \Xi^{0} \bar{\Xi}^{0}, \Xi^{-} \bar{\Xi}^{+}\right\}$.

So that, the minimization has been performed with respect to the six free parameters of the set $\xi$, by exploiting eight experimental constraints, that are reported in Table II. The obtained $\mathrm{BRs}, \mathrm{BR}_{\mathcal{B} \overline{\mathcal{B}}}^{\text {th }}$, with $\mathcal{B} \overline{\mathcal{B}} \in \Omega$, are given by the combinations of these parameters reported in Table I, following the expression

$$
\mathrm{BR}_{\mathcal{B B}}=\frac{|\vec{p}|}{8 \pi M_{\psi(2 S)}^{2} \Gamma_{\psi(2 S)}}\left|\mathcal{A}_{\mathcal{B} \overline{\mathcal{B}}}^{g g g}+\mathcal{A}_{\mathcal{B} \overline{\mathcal{B}}}^{g g \gamma}+\mathcal{A}_{\mathcal{B} \overline{\mathcal{B}}}^{\gamma}\right|^{2}
$$

where $\vec{p}$ is the three-momentum of the baryon in the $\mathcal{B} \overline{\mathcal{B}}$ center of mass frame, and $\Gamma_{\psi(2 S)}$ is the width of the $\psi(2 S)$ meson.

TABLE II. Branching ratios data from PDG [1]. The last row is from Eq. (4), while the sixth one is from BESIII [15].

\begin{tabular}{lcc}
\hline \hline Decay process & Branching ratio & Error \\
\hline$\psi(2 S) \rightarrow p \bar{p}$ & $(2.94 \pm 0.08) \times 10^{-4}$ & $2.72 \%$ \\
$\psi(2 S) \rightarrow n \bar{n}$ & $(3.06 \pm 0.15) \times 10^{-4}$ & $4.90 \%$ \\
$\psi(2 S) \rightarrow \Lambda \bar{\Lambda}$ & $(3.81 \pm 0.13) \times 10^{-4}$ & $3.41 \%$ \\
$\psi(2 S) \rightarrow \Sigma^{+} \bar{\Sigma}^{-}$ & $(2.32 \pm 0.12) \times 10^{-4}$ & $5.17 \%$ \\
$\psi(2 S) \rightarrow \Sigma^{0} \bar{\Sigma}^{0}$ & $(2.35 \pm 0.09) \times 10^{-4}$ & $3.83 \%$ \\
$\psi(2 S) \rightarrow \Xi^{0} \bar{\Xi}^{0}$ & $(2.73 \pm 0.13) \times 10^{-4}$ & $4.76 \%$ \\
$\psi(2 S) \rightarrow \Xi^{-} \bar{\Xi}^{+}$ & $(2.87 \pm 0.11) \times 10^{-4}$ & $3.83 \%$ \\
$\psi(2 S) \rightarrow \gamma \rightarrow p \bar{p}$ & $(1.35 \pm 0.07) \times 10^{-6}$ & $5.19 \%$ \\
\hline \hline
\end{tabular}


TABLE III. Best values of the parameters, for the $\psi(2 S)$ meson, describing the decay $\mathcal{B} \overline{\mathcal{B}}$ amplitudes, see Table I, obtained by minimizing the $\chi^{2}$ defined in Eq. (6), using the data reported in Table II. The third row is from Eq. (5).

\begin{tabular}{lc}
\hline \hline$\chi^{2} / N_{\text {dof }}$ & 6.85 \\
\hline$G_{0}$ & $(4.508 \pm 0.052) \times 10^{-3} \mathrm{GeV}$ \\
$D_{e}$ & $(1.25 \pm 0.07) \times 10^{-4} \mathrm{GeV}$ \\
$D_{m}$ & $(-2.30 \pm 0.36) \times 10^{-4} \mathrm{GeV}$ \\
$F_{e}$ & $(1.65 \pm 0.17) \times 10^{-4} \mathrm{GeV}$ \\
$F_{m}$ & $(-2.40 \pm 0.60) \times 10^{-4} \mathrm{GeV}$ \\
$\varphi$ & $1.10 \pm 0.54=(63 \pm 31)^{\circ}$ \\
$R$ & $(-5.9 \pm 2.6) \times 10^{-2}$ \\
\hline \hline
\end{tabular}

\section{RESULTS AND DISCUSSION}

The results of the $\chi^{2}$ minimization are summarized in Table III, the errors have been obtained by means of a Monte Carlo procedure. The results obtained by performing the same analysis in the case of the $J / \psi$ meson are reported for comparison in Table IV. Instead, Table V reports input and output values, together with their discrepancies in units of standard deviations. ${ }^{1}$ Such a comparison is quite useful to clearly identify the main contributions to the $\chi^{2}$, and hence the channels whose dynamics seems to escape the phenomenological description provided by the model. The value that has been obtained for the BR of the decay $\psi(2 S) \rightarrow \Sigma^{-} \bar{\Sigma}^{+}$, which is still unobserved, represents a prediction of the model.

The minimum of the $\chi^{2}$, normalized to the number of degrees of freedom $N_{\text {dof }}$, is

$$
\frac{\chi^{2}\left(\xi^{\text {best }}\right)}{N_{\text {dof }}}=6.85
$$

where $N_{\text {dof }}=N_{\text {const }}-N_{\text {param }}=2$, having eight constraints, $N_{\text {const }}=8$, and six free parameters, $N_{\text {param }}=6$.

The obtained value of the ratio $R=-0.059 \pm 0.026$, last row of Table III, can be compared with that obtained for the $J / \psi$ meson, $R_{J / \psi}=-0.097 \pm 0.021$, last row of Table IV, as well as with the asymptotic QCD prediction ${ }^{2}$ of Eq. (2), $R_{\mathrm{pQCD}} \sim-0.024$.

\footnotetext{
${ }^{1}$ As usually we define the discrepancy between two quantities with given errors $x \pm \sigma_{x}$ and $y \pm \sigma_{y}$ as

$$
\operatorname{Discr}(\sigma)=|x-y| / \sqrt{\sigma_{x}^{2}+\sigma_{y}^{2}} .
$$

${ }^{2}$ This value has been obtained by using Eq. (2) with $\alpha_{S}\left(M_{J / \psi}^{2}\right)=\left|\alpha_{s}^{\mathrm{pQCD}}\left(-M_{J / \psi}^{2}\right)\right|$, where $\alpha_{s}^{\mathrm{pQCD}}\left(Q^{2}\right)$ is given in Eq. (9) of Ref. [16] with the higher order coefficients $\beta_{2}$ and $\beta_{3}$ obtained in the $\overline{\mathrm{MS}}$ renormalization scheme and $\Lambda_{\mathrm{QCD}}=0.35 \mathrm{GeV}$.
}

TABLE IV. Best parameters obtained for the $J / \psi$ meson [2].

\begin{tabular}{lc}
\hline \hline$\chi^{2} / N_{\text {dof }}$ & 1.33 \\
\hline$G_{0}^{J / \psi}$ & $(5.73511 \pm 0.0059) \times 10^{-3} \mathrm{GeV}$ \\
$D_{e}^{J / \psi}$ & $(4.52 \pm 0.19) \times 10^{-4} \mathrm{GeV}$ \\
$D_{m}^{J / \psi}$ & $(-3.74 \pm 0.34) \times 10^{-4} \mathrm{GeV}$ \\
$F_{e}^{J / \psi}$ & $(7.91 \pm 0.62) \times 10^{-4} \mathrm{GeV}$ \\
$F_{m}^{J / \psi}$ & $(2.42 \pm 0.12) \times 10^{-4} \mathrm{GeV}$ \\
$\varphi_{J / \psi}$ & $1.27 \pm 0.14=(73 \pm 8)^{\circ}$ \\
$R_{J / \psi}$ & $(-9.7 \pm 2.1) \times 10^{-2}$ \\
\hline \hline
\end{tabular}

TABLE V. Input and output values of the BRs and their discrepancies, for the $\psi(2 S)$ meson.

\begin{tabular}{lccc}
\hline \hline $\mathcal{B} \overline{\mathcal{B}}$ & $\mathrm{BR}_{\mathcal{B} \overline{\mathcal{B}}}^{\exp } \times 10^{4}$ & $\mathrm{BR}_{\mathcal{B} \overline{\mathcal{B}}} \times 10^{4}$ & Discr. $(\sigma)$ \\
\hline$p \bar{p}$ & $2.94 \pm 0.08$ & $3.02 \pm 0.26$ & 0.294 \\
$n \bar{n}$ & $3.06 \pm 0.15$ & $3.04 \pm 0.19$ & 0.083 \\
$\Lambda \bar{\Lambda}$ & $3.81 \pm 0.13$ & $3.53 \pm 0.15$ & 1.411 \\
$\Sigma^{+} \bar{\Sigma}^{-}$ & $2.32 \pm 0.12$ & $2.19 \pm 0.20$ & 0.557 \\
$\Sigma^{0} \bar{\Sigma}^{0}$ & $2.35 \pm 0.09$ & $2.36 \pm 0.12$ & 0.067 \\
$\Xi^{0} \bar{\Xi}^{0}$ & $2.73 \pm 0.13$ & $3.05 \pm 0.17$ & 1.495 \\
$\Xi^{-} \bar{\Xi}^{+}$ & $2.87 \pm 0.11$ & $2.79 \pm 0.18$ & 0.379 \\
$\Sigma^{-} \bar{\Sigma}^{+}$ & $\ldots$ & $2.01 \pm 0.14$ & $\ldots$ \\
$\gamma \rightarrow p \bar{p}$ & $0.0135 \pm 0.0007$ & $0.0133 \pm 0.0007$ & $\sim 0$ \\
\hline \hline
\end{tabular}

Meanwhile, the three contributions, purely strong, purely EM and mixed strong-EM, to the total BR are listed in Table VI. For a comparison, the corresponding values obtained for the $J / \psi$ meson are reported in Table VII.

\section{A. Further hypothesis}

The minimization procedure has provided a quite large $\chi^{2}$ minimum value, see Eq. (8), in particular, it is larger than the one obtained by performing the same analysis for the $J / \psi$ meson, whose value is shown in the first row of Table IV.

By assuming the reliability of all the data, a so different capability of the model to describe the dynamics underlying the decays into the same channels of the two similar

TABLE VI. Strong (second column), EM (third column) and mixed (fourth column) BRs for the $\psi(2 S)$ meson.

\begin{tabular}{lccc}
\hline \hline $\mathcal{B} \overline{\mathcal{B}}$ & $\mathrm{BR}_{\mathcal{B} \overline{\mathcal{B}}}^{g g g} \times 10^{4}$ & $\mathrm{BR}_{\mathcal{B} \overline{\mathcal{B}}}^{\gamma} \times 10^{6}$ & $\mathrm{BR}_{\mathcal{B} \overline{\mathcal{B}}}^{g g \gamma} \times 10^{6}$ \\
\hline$p \bar{p}$ & $3.20 \pm 0.12$ & $1.34 \pm 0.17$ & $1.3 \pm 1.0$ \\
$n \bar{n}$ & $3.20 \pm 0.12$ & $0.99 \pm 0.11$ & 0 \\
$\Lambda \bar{\Lambda}$ & $3.61 \pm 0.13$ & $0.229 \pm 0.026$ & 0 \\
$\Sigma^{+} \bar{\Sigma}^{-}$ & $2.30 \pm 0.10$ & $1.19 \pm 0.15$ & $0.94 \pm 0.73$ \\
$\Sigma^{-} \bar{\Sigma}^{+}$ & $2.29 \pm 0.10$ & $0.028 \pm 0.022$ & $0.94 \pm 0.73$ \\
$\Sigma^{0} \bar{\Sigma}^{0}$ & $2.30 \pm 0.10$ & $0.219 \pm 0.024$ & 0 \\
$\Xi^{0} \bar{\Xi}^{0}$ & $3.19 \pm 0.11$ & $0.807 \pm 0.089$ & 0 \\
$\Xi^{-} \bar{\Xi}^{+}$ & $3.17 \pm 0.11$ & $0.026 \pm 0.020$ & $1.28 \pm 0.98$ \\
\hline \hline
\end{tabular}


TABLE VII. Strong (second column), EM (third column) and mixed (fourth column) BRs for the $J / \psi$ meson [2].

\begin{tabular}{lccc}
\hline \hline $\mathcal{B} \overline{\mathcal{B}}$ & $\mathrm{BR}_{\mathcal{B} \overline{\mathcal{B}}}^{g g g} \times 10^{3}$ & $\mathrm{BR}_{\mathcal{B} \overline{\mathcal{B}}}^{\gamma} \times 10^{5}$ & $\mathrm{BR}_{\mathcal{B} \overline{\mathcal{B}}}^{g g \gamma} \times 10^{5}$ \\
\hline$p \bar{p}$ & $2.220 \pm 0.085$ & $8.52 \pm 0.89$ & $2.19 \pm 0.93$ \\
$n \bar{n}$ & $2.220 \pm 0.085$ & $4.50 \pm 0.38$ & 0 \\
$\Lambda \bar{\Lambda}$ & $2.020 \pm 0.042$ & $0.981 \pm 0.083$ & 0 \\
$\Sigma^{+} \bar{\Sigma}^{-}$ & $1.100 \pm 0.030$ & $6.86 \pm 0.72$ & $1.08 \pm 0.46$ \\
$\Sigma^{-} \bar{\Sigma}^{+}$ & $1.090 \pm 0.030$ & $0.52 \pm 0.20$ & $1.07 \pm 0.46$ \\
$\Sigma^{0} \bar{\Sigma}^{0}$ & $1.100 \pm 0.030$ & $0.902 \pm 0.076$ & 0 \\
$\Xi^{0} \bar{\Xi}^{0}$ & $1.260 \pm 0.053$ & $2.99 \pm 0.25$ & 0 \\
$\Xi^{-} \bar{\Xi}^{+}$ & $1.240 \pm 0.052$ & $0.43 \pm 0.16$ & $1.22 \pm 0.52$ \\
\hline \hline
\end{tabular}

vector charmonia, the leading and the first excited states, $J / \psi$ and $\psi(2 S)$, could be ascribed to the presence of some contribution, negligible at the $J / \psi$ mass, but having an effect at the $\psi(2 S)$ mass, that is not included in the original Lagrangian density of Eq. (1).

To verify the eventuality that the missing contribution concerned a single $\mathcal{B} \overline{\mathcal{B}}$ decay channel, we minimize

$$
\chi_{\not p}^{2}=\sum_{\mathcal{B} \overline{\mathcal{B}} \in \Omega \backslash\{\mathcal{B} \overline{\mathcal{B}}\}}\left(\frac{\mathrm{BR}_{\mathcal{B} \overline{\mathcal{B}}}^{\mathrm{th}}-\mathrm{BR}_{\mathcal{B} \overline{\mathcal{B}}}^{\mathrm{exp}}}{\delta \mathrm{BR}_{\mathcal{B} \overline{\mathcal{B}}}^{\mathrm{exp}}}\right)^{2},
$$

that includes all the experimental constraint with the exception of the $\mathcal{B} \overline{\mathcal{B}}$ one, and hence it can be seen as a discrete function of the channel $\mathcal{B} \overline{\mathcal{B}}$, which varies in set $\Omega$ given in Eq. (7). The behavior of $\chi_{\not p}^{2}$ versus the decay channel $\mathcal{B} \overline{\mathcal{B}}$, is shown, in logarithmic scale, in Fig. 2.

We observe that at $\mathcal{B} \overline{\mathcal{B}}=\Lambda \bar{\Lambda}$, i.e., by excluding the datum on the BR of the $\Lambda \bar{\Lambda}$ final state, the discrete function $\chi_{\not p}^{2}$ reaches its minimum value, which is one order of magnitude smaller than the value of Eq. (8), obtained by taking into account all the available data.

The simplest explanation for such a result is that there should be some particular mechanism that enhances the

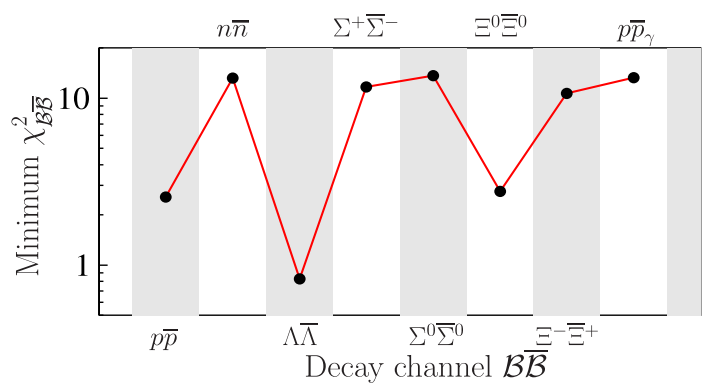

FIG. 2. Comparison of $\chi_{\not b}^{2}$ values obtained with the minimization procedure of Eq. (6), by using the data reported in Table II, but excluding, each time, an experimental datum. The channel of the excluded datum is reported on the abscissa. For each case $N_{\text {dof }}=1$. affinity between the $\psi(2 S)$ and the $\Lambda \bar{\Lambda}$ final state, by favoring the decay $\psi(2 S) \rightarrow \Lambda \bar{\Lambda}$ with respect to the $J / \psi \rightarrow \Lambda \bar{\Lambda}$, that is not considered in our model. Unfortunately, it is quite difficult envisaging the nature of the eventual missing piece of the Lagrangian density, by only relying on its incapability to describe the decay into $\Lambda \bar{\Lambda}$, once all the other have been considered.

In the light of that, the $\Lambda \bar{\Lambda}$ channel has been excluded from the set of the experimental constraints, and hence the minimization procedure has been based on the function $\chi_{\alpha,}^{2}$, obtained from Eq. (9) with $\mathcal{B} \overline{\mathcal{B}}=\Lambda \bar{\Lambda}$. The coupling constants, the relative phase $\varphi$, and the ratio $R$ obtained in this case are reported in Table VIII.

We notice that, even though the $\chi_{\not p}^{2}$ has three minima of apparently the same order, i.e., besides the one of the $\Lambda \bar{\Lambda}$ channel, there are those corresponding to $p \bar{p}$ and $\Xi^{0} \bar{\Xi}^{0}$, they are crucially different. While $\chi_{\not \alpha}^{2}$ is lower than one, $\chi_{\ddagger}^{2}$ and $\chi_{\not}^{2}$ remain larger than two. These values, even on the light of the only one degree of freedom, disfavor the hypotheses of excluding the corresponding BR data.

A difference very interesting and meaningful, as we will see in the following discussion, among the two sets of parameters that minimize the complete $\chi^{2}$ of Eq. (6), reported in Table III, and $\chi_{\neq}^{2}$, is the outcome for the ratio $R$, that passes from the negative value $R=(-5.9 \pm 2.6) \times$ $10^{-2}$ to a positive value but compatible with zero $R=(2.3 \pm 3.4) \times 10^{-2}$. We interpreted this result as a phenomenological manifestation of the tendency toward a perturbative regime of the underlying QCD dynamics. Indeed, in this case, the ratio $R$ would acquire a dependence on the baryon electric charge $Q_{\mathcal{B}}$, as shown in Eq. (2).

This is a quite natural consequence of the direct computation of the $g g \gamma$ amplitude, whose Feynman diagram is shown in the lower panel of Fig. 1, by summing up the three possible contributions where the photon couples to each of the three valence-quark lines. Since each

TABLE VIII. Best values of the parameters, for the $\psi(2 S)$ meson, describing the decay $\mathcal{B} \overline{\mathcal{B}}$ amplitudes, see Table I, obtained by minimizing the $\chi^{2}$ defined in Eq. (6), using the data reported in Table II with the exclusion of the $\Lambda \bar{\Lambda}$ final state. The third row is from Eq. (5).

\begin{tabular}{lc}
\hline \hline$\chi_{\alpha}^{2} / N_{\mathrm{dof}}$ & 0.83 \\
\hline$G_{0}$ & $(4.331 \pm 0.071) \times 10^{-3} \mathrm{GeV}$ \\
$D_{e}$ & $(1.25 \pm 0.07) \times 10^{-4} \mathrm{GeV}$ \\
$D_{m}$ & $(-1.11 \pm 0.44) \times 10^{-4} \mathrm{GeV}$ \\
$F_{e}$ & $(1.66 \pm 0.17) \times 10^{-4} \mathrm{GeV}$ \\
$F_{m}$ & $(-1.35 \pm 0.67) \times 10^{-4} \mathrm{GeV}$ \\
$\varphi$ & $1.97 \pm 0.53=(111 \pm 30)^{\circ}$ \\
$R$ & $(2.3 \pm 3.4) \times 10^{-2}$ \\
\hline \hline
\end{tabular}


TABLE IX. Best values of the parameters, for the $\psi(2 S)$ meson, describing the decay $\mathcal{B} \overline{\mathcal{B}}$ amplitudes, see Table I ( ${ }^{*}$ with opposite $R$ for the negative final state baryons), from the $\chi_{\alpha}^{2}$ minimization and considering a double-sign ratio $R$. The third row is from Eq. (5).

\begin{tabular}{lc}
\hline \hline$\chi_{\not,}^{2} / N_{\mathrm{dof}}$ & 0.17 \\
\hline$G_{0}$ & $(4.374 \pm 0.039) \times 10^{-3} \mathrm{GeV}$ \\
$D_{e}$ & $(1.25 \pm 0.07) \times 10^{-4} \mathrm{GeV}$ \\
$D_{m}$ & $(-1.35 \pm 0.28) \times 10^{-4} \mathrm{GeV}$ \\
$F_{e}$ & $(1.67 \pm 0.17) \times 10^{-4} \mathrm{GeV}$ \\
$F_{m}$ & $(-1.17 \pm 0.69) \times 10^{-4} \mathrm{GeV}$ \\
$\varphi$ & $1.52 \pm 0.25=(87 \pm 15)^{\circ}$ \\
$R^{*}$ & $(-2.2 \pm 2.0) \times 10^{-2}$ \\
\hline \hline
\end{tabular}

coupling is weighted by the quark electric charge, the total contribution turns out to be proportional to the baryon electric charge.

The reason why the agreement with zero of a unique value of $R$ can be interpreted as a phenomenological requirement of $\mathrm{pQCD}$ restoration relies on the assumption of an almost linear response of the model to the experimental constraints. In the sense that, if we force two free parameters, supposed to be opposite, to have instead a common value, the minimization procedure would give for such a parameter a value compatible with their mean and hence close to zero.

In light of that, we have considered the free parameter $R$ as having the same modulus for all decay channels but with sign linked to that of the baryon electric charge, in particular, as predicted by $\mathrm{pQCD}, R$ turns out to be negative for positive charges, see Eq. (2).

In more detail, the model has been modified by considering two opposite values for the ratio $R$, namely: $R=-|R|$ for $p$ and $\Sigma^{+} ; R=|R|$ for $\Sigma^{-}$and $\Xi^{-}$, actually in this case only the latter did affect the minimization procedure since there is no data on the BR of the decay $\psi(2 S) \rightarrow \Sigma^{-} \bar{\Sigma}^{+}$.

The lower value for the minimum of $\chi_{\not k}^{2}$, decreasing from 0.83 with a single-sign $R$ down to 0.17 with a double-sign $R$, see Tables VIII and IX, and mainly the good agreement with the pQCD expectation from Eq. $(2)^{3}$

$$
R_{\mathrm{pQCD}}\left(M_{\psi(2 S)}^{2}\right) \simeq-0.025
$$

did demonstrate the goodness of this hypothesis, even though the best value of the ratio $R$ is still in agreement with zero, meaning that the $g g \gamma$ contribution in the $\psi(2 S)$ case plays a role not so important as it does for the $J / \psi$.

The differences among the best values of the parameters obtained including the $\Lambda \bar{\Lambda} \mathrm{BR}$, with a single-sign $R$, and those without the $\Lambda \bar{\Lambda}$ constraint, but considering a doublesign, QCD-inspired ratio $R$, reported in Tables III and IX,

\footnotetext{
${ }^{3}$ See footnote 2 .
}

respectively, are mainly related to the parameters $D_{m}$ and $F_{m}$, responsible for the $\mathrm{SU}(3)$ symmetry breaking due to the $(u, d)-s$ quark mass difference, whose moduli, in the second case are reduced by a factor of almost two with respect to the first case. This is not unexpected, in fact, in the framework of $\mathrm{pQCD}$, a reduction of such effects, i.e., a tendency toward the flavor SU(3)-symmetry restoration appears quite natural.

\section{CONCLUSIONS}

The $\psi(2 S)$ decays into spin-1/2 baryons belonging to the SU(3) octet have been studied in the framework of the same phenomenological model used for the $J / \psi$ meson [2]. Despite the natural affinity between the two lightest vector charmonia, the model seems to fail in providing a complete description of the decay mechanisms in the case of the $\psi(2 S)$. In particular, the $\Lambda \bar{\Lambda}$ decay channel has a BR that exceeds the value that the model can provide, by highlighting the eventual, or, at least, phenomenologically required, presence of an additional contribution especially effective in this peculiar decay channel.

The investigation of a possible $\Lambda$-enhancing mechanism goes beyond the scope of this work so that, we only limited ourselves to notice such a phenomenon and act accordingly by excluding the corresponding experimental constraint from the analysis. It follows that the main results of this study have been those obtained by do not considering the $\Lambda \bar{\Lambda}$ channel.

The main difference between the findings of the studies on the $J / \psi$ and $\psi(2 S)$ decays into baryon-antibaryon pairs has consisted in the role played by the couplings $D_{m}$ and $F_{m}$, see Tables III and IV, that account for the SU(3) symmetry breaking due to quark mass differences.

In particular, these parameters have the same sign in the case of the $\psi(2 S)$, while they have a different sign in the case of the $J / \psi$. Similar differences, due to the $S U(3)$ breaking terms, have also been observed by studying the angular distributions of the decays into the $\Sigma$ and the peculiar $\Lambda$ channel $[4,5]$. One of the causes of such different behaviors could be the $\Lambda$-enhancing mechanism previously discussed. Unfortunately, the effective Lagrangian model, used here, provides coupling constants that, in the language of "strong electric and magnetic form factors", $g_{E, M}^{\mathcal{B}, \psi}[4,5]$, for the decay of the charmonium $\psi$ in to the $\mathcal{B} \overline{\mathcal{B}}$ baryon pair, could be considered as a unique effective coupling constant, whose modulus squared is given by the combination: $\left|g^{\mathcal{B}, \psi}\right|^{2}=$ $\left|g_{M}^{\mathcal{B}, \psi}\right|^{2}+2 M_{B}^{2}\left|g_{E}^{\mathcal{B}, \psi}\right|^{2} / M_{\psi(2 S)}^{2}$. This means that the information on the angular distributions cannot easily be used.

Nevertheless, we are exploring the possibility of exploiting the value of the polarization parameter

$$
\alpha^{\mathcal{B}, \psi}=\frac{\left|g_{M}^{\mathcal{B}, \psi}\right|^{2}-4 M_{B}^{2}\left|g_{E}^{\mathcal{B}, \psi}\right|^{2} / M_{\psi(2 S)}^{2}}{\left|g_{M}^{\mathcal{B}, \psi}\right|^{2}+4 M_{B}^{2}\left|g_{E}^{\mathcal{B}, \psi}\right|^{2} / M_{\psi(2 S)}^{2}},
$$




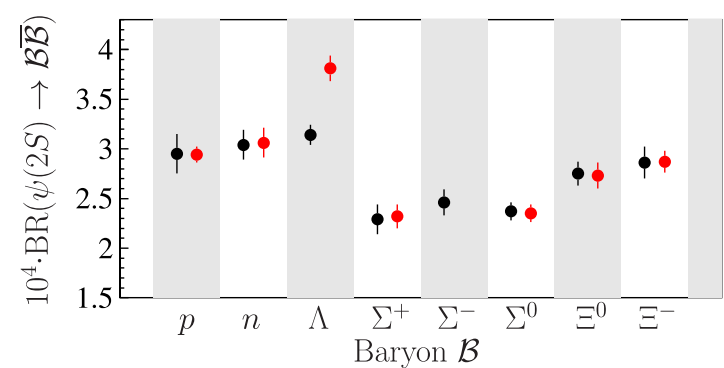

FIG. 3. Comparison of BRs (experimental input vs model predictions). The red points are from Table II, while the black ones, from Table XI, are the corresponding values obtained as outcomes of the minimization of $\chi_{\not, \alpha}^{2}$ and considering a doublesign ratio $R$. The errors are obtained by means of a Monte Carlo procedure.

obtained by studying the $\mathcal{B} \overline{\mathcal{B}}$ angular distribution in the process $e^{+} e^{-} \rightarrow \psi \rightarrow \mathcal{B} \overline{\mathcal{B}}$, in combination with the modulus squared of the corresponding effective coupling constant $g^{\mathcal{B}, \psi}$, to achieve more information on the strong form factors and hence on the dynamics of the charmonium decays in to baryon pairs [17].

Even the couplings accounting for the EM SU(3) symmetry breaking, $D_{e}$ and $F_{e}$, in the case of $\psi(2 S)$ are smaller (compared to the dominant contribution $G_{0}$ ) than those of the $J / \psi$.

The BRs obtained by the $\chi_{\alpha}^{2}$ minimization procedure and considering a double-sign ratio $R$, whose single contributions are reported in Table $\mathrm{X}$, are in agreement, at most within 0.16 sigmas, apart from that of the $\Lambda \bar{\Lambda}$ channel excluded from the $\chi^{2}$ function, with the corresponding input values, as reported in Table XI and shown in Fig. 3. The BR of the unobserved decay $\psi(2 S) \rightarrow \Sigma^{-} \bar{\Sigma}^{+}$, i.e., from Table XI, $\mathrm{BR}_{\Sigma^{-} \bar{\Sigma}^{+}}=(2.46 \pm 0.13) \times 10^{-4}$ represents a prediction of the model. Moreover, the strong-EM relative phase $\varphi=(87 \pm 15)^{\circ}$ is in agreement with the phase of the $J / \psi, \varphi_{J / \psi}=(73 \pm 8)^{\circ}$.

TABLE X. Strong (second column), EM (third column), and mixed (fourth column) BRs for the $\psi(2 S)$ meson, from the $\chi_{\alpha}^{2}$ minimization and by considering a double-sign ratio $R$.

\begin{tabular}{lccc}
\hline \hline $\mathcal{B} \overline{\mathcal{B}}$ & $\mathrm{BR}_{\mathcal{B} \overline{\mathcal{B}}}^{g g g} \times 10^{4}$ & $\mathrm{BR}_{\mathcal{B} \overline{\mathcal{B}}}^{\gamma} \times 10^{6}$ & $\mathrm{BR}_{\mathcal{B} \overline{\mathcal{B}}}^{g g \gamma} \times 10^{6}$ \\
\hline$p \bar{p}$ & $3.05 \pm 0.11$ & $1.36 \pm 0.17$ & $0.30 \pm 0.37$ \\
$n \bar{n}$ & $3.05 \pm 0.11$ & $0.99 \pm 0.11$ & 0 \\
$\Lambda \bar{\Lambda}$ & $3.150 \pm 0.094$ & $0.229 \pm 0.025$ & 0 \\
$\Sigma^{+} \bar{\Sigma}^{-}$ & $2.36 \pm 0.08$ & $1.20 \pm 0.15$ & $0.23 \pm 0.29$ \\
$\Sigma^{-} \bar{\Sigma}^{+}$ & $2.35 \pm 0.08$ & $0.029 \pm 0.022$ & $0.23 \pm 0.28$ \\
$\Sigma^{0} \bar{\Sigma}^{0}$ & $2.36 \pm 0.08$ & $0.219 \pm 0.024$ & 0 \\
$\Xi^{0} \bar{\Xi}^{0}$ & $2.75 \pm 0.10$ & $0.806 \pm 0.089$ & 0 \\
$\Xi^{-} \bar{\Xi}^{+}$ & $2.74 \pm 0.10$ & $0.027 \pm 0.020$ & $0.27 \pm 0.34$ \\
\hline \hline
\end{tabular}

TABLE XI. Input and output values of the BRs and their discrepancies, for the $\psi(2 S)$ meson, from the $\chi_{\not \alpha}^{2}$ minimization and considering a double-sign ratio $R$.

\begin{tabular}{lccc}
\hline \hline $\mathcal{B} \overline{\mathcal{B}}$ & $\mathrm{BR}_{\mathcal{B} \overline{\mathcal{B}}}^{\exp } \times 10^{4}$ & $\mathrm{BR}_{\mathcal{B \mathcal { B }}} \times 10^{4}$ & Discr. $(\sigma)$ \\
\hline$p \bar{p}$ & $2.94 \pm 0.08$ & $2.95 \pm 0.20$ & 0.046 \\
$n \bar{n}$ & $3.06 \pm 0.15$ & $3.04 \pm 0.15$ & 0.094 \\
$\Lambda \bar{\Lambda}$ & $3.81 \pm 0.13$ & $3.14 \pm 0.10$ & 4.1 \\
$\Sigma^{+} \bar{\Sigma}^{-}$ & $2.32 \pm 0.12$ & $2.29 \pm 0.15$ & 0.16 \\
$\Sigma^{-} \bar{\Sigma}^{+}$ & $\ldots$ & $2.46 \pm 0.13$ & $\ldots$ \\
$\Sigma^{0} \bar{\Sigma}^{0}$ & $2.35 \pm 0.09$ & $2.37 \pm 0.09$ & 0.14 \\
$\Xi^{0} \bar{\Xi}^{0}$ & $2.73 \pm 0.13$ & $2.75 \pm 0.12$ & 0.11 \\
$\Xi^{-} \bar{\Xi}^{+}$ & $2.87 \pm 0.11$ & $2.86 \pm 0.16$ & 0.052 \\
\hline \hline
\end{tabular}

In the case of the $\psi(2 S)$, the ratio $R$ became a key parameter, in fact, it can be used to reveal the QCD regime. By studying its values obtained as a consequence of different hypotheses, primarily the exclusion of the $\Lambda \bar{\Lambda}$ channel from the set of the experimental constraints guided us toward a better understanding of the dynamics of these decays. The really interesting conclusion is that, already at $\psi(2 S)$ mass, the QCD regime is perturbative. This means that the expression of the ratio $R$ is known, as given in Eq. (2), it is proportional to the ratio between the finestructure constant and the strong running coupling constant. Moreover, its sign varies accordingly to that of the electric charge of the baryon, or better, by including also the case of the null value for neutral baryons, we can say that it is proportional to baryon electric charge. The hypothesis of perturbative regime of the QCD is supported, not only by the goodness of the description in terms of a charge-dependent ratio $R$, but mainly by the numerical value of this parameter, reported in Table IX, that, despite the large error, has a central value in good agreement with the pQCD expectation given in Eq. (10).

As already done in the case of the $J / \psi$ meson, for the first time, the strong, the EM and the mixed strong-EM contributions to the total $\mathrm{BR}$ of the decays $\psi(2 S) \rightarrow \mathcal{B \mathcal { B }}$ have been separated. The obtained values reported in Table X can be compared to those of the $J / \psi$ meson in Table VII.

It is evident that the purely strong contribution to the total BR is larger in the case of the $J / \psi$ with respect to the $\psi(2 S)$. This behavior is compatible with the decreasing trend of the QCD running coupling constant as $q^{2}$ increases, from the $J / \psi$ to the $\psi(2 S)$ mass.

The EM contributions for the $\psi(2 S)$ meson, again from Table $X$, follow a hierarchy with decreasing values of orders of magnitude. In fact for the baryon pairs $p \bar{p}, n \bar{n}$, $\Sigma^{+} \bar{\Sigma}^{-}$, and $\Xi^{0} \bar{\Xi}^{0}$ we have $\mathrm{BR}^{\gamma} \sim 10^{-6}$, for $\Sigma^{0} \bar{\Sigma}^{0}$ and $\Lambda \bar{\Lambda}$ we have $\mathrm{BR}^{\gamma} \sim 10^{-7}$ and, finally, for the two pairs $\Sigma^{-} \bar{\Sigma}^{+}$and $\Xi^{-} \bar{\Xi}^{+}$we have $\mathrm{BR}^{\gamma} \sim 10^{-8}$. A similar trend, but with one order of magnitude more, can be seen also in the case of the $J / \psi$ meson, see Table VII. 
TABLE XII. Nonresonant $e^{+} e^{-} \rightarrow \mathcal{B} \overline{\mathcal{B}}$ Born cross sections at $q^{2}=M_{\psi(2 S)}^{2}$.

\begin{tabular}{lc}
\hline \hline$e^{+} e^{-} \rightarrow \mathcal{B} \overline{\mathcal{B}}$ & Cross section at the $q^{2}=M_{\psi(2 S)}^{2}$ \\
\hline$e^{+} e^{-} \rightarrow \Sigma^{0} \bar{\Sigma}^{0}$ & $(0.175 \pm 0.024) \mathrm{pb}$ \\
$e^{+} e^{-} \rightarrow \Lambda \bar{\Lambda}$ & $(0.183 \pm 0.024) \mathrm{pb}$ \\
$e^{+} e^{-} \rightarrow\left(\Lambda \bar{\Sigma}^{0}+\right.$ c.c. $)$ & $(0.538 \pm 0.073) \mathrm{pb}$ \\
$e^{+} e^{-} \rightarrow p \bar{p}$ & $(1.10 \pm 0.16) \mathrm{pb}$ \\
$e^{+} e^{-} \rightarrow n \bar{n}$ & $(0.79 \pm 0.11) \mathrm{pb}$ \\
$e^{+} e^{-} \rightarrow \Sigma^{+} \bar{\Sigma}^{-}$ & $(0.97 \pm 0.14) \mathrm{pb}$ \\
$e^{+} e^{-} \rightarrow \Sigma^{-} \bar{\Sigma}^{+}$ & $(0.024 \pm 0.018) \mathrm{pb}$ \\
$e^{+} e^{-} \rightarrow \Xi^{0} \bar{\Xi}^{0}$ & $(0.645 \pm 0.086) \mathrm{pb}$ \\
$e^{+} e^{-} \rightarrow \Xi^{-} \bar{\Xi}^{+}$ & $(0.022 \pm 0.017) \mathrm{pb}$ \\
\hline \hline
\end{tabular}

We use the purely EM BR, depending only on the parameters $D_{e}$ and $F_{e}$, to calculate the Born nonresonant cross section of the annihilation processes $e^{+} e^{-} \rightarrow \mathcal{B B}$ at the $\psi(2 S)$ mass. The results are reported in Table XII. They appear independent on the $\Lambda \bar{\Lambda} \mathrm{BR}$, indeed, as can be seen in Tables III and VIII, the values of $D_{e}$ and $F_{e}$, obtained including and not including, respectively, the datum on the $\Lambda \bar{\Lambda} \mathrm{BR}$, are almost the same.

\section{ACKNOWLEDGMENTS}

We would like to warmly acknowledge the Italian group of the BESIII Collaboration for the useful and fruitful discussions on experimental and phenomenological aspects concerning $J / \psi$ decays. This work was supported in part by the STRONG-2020 project of the European Union's Horizon 2020 research and innovation programme under Grant Agreement No. 824093.
[1] M. Tanabashi et al. (Particle Data Group), Phys. Rev. D 98, 030001 (2018).

[2] R. Baldini Ferroli, A. Mangoni, S. Pacetti, and K. Zhu, Phys. Lett. B 799, 135041 (2019).

[3] R. Baldini Ferroli, A. Mangoni, and S. Pacetti, Phys. Rev. C 98, 045210 (2018).

[4] M. Ablikim et al. (BESIII Collaboration), Phys. Rev. D 95, 052003 (2017).

[5] M. Alekseev et al., Chin. Phys. C 43, 023103 (2019).

[6] K. Zhu, X. H. Mo, and C. Z. Yuan, Int. J. Mod. Phys. A 30, 1550148 (2015).

[7] V. Chernyak and I. Zhitnitsky, Nucl. Phys. B246, 52 (1984).

[8] M. Claudson, S. L. Glashow, and M. B. Wise, Phys. Rev. D 25, 1345 (1982).

[9] J. G. Korner, Z. Phys. C 33, 529 (1987).
[10] B. Aubert et al. (BABAR Collaboration), Phys. Rev. D 73, 012005 (2006).

[11] M. Ablikim et al. (BESIII Collaboration), Phys. Rev. D 99, 092002 (2019).

[12] V. A. Matveev, R. M. Muradyan, and A. N. Tavkhelidze, Teor. Mat. Fiz. 15, 332 (1973).

[13] S. J. Brodsky and G. R. Farrar, Phys. Rev. Lett. 31, 1153 (1973).

[14] R. Baldini Ferroli, A. Mangoni, and S. Pacetti, Eur. Phys. J. C 80, 903 (2020).

[15] M. Ablikim et al. (BESIII Collaboration), Phys. Lett. B 770, 217 (2017).

[16] A. Deur, S. J. Brodsky, and G. F. de Teramond, Phys. Lett. B 757, 275 (2016).

[17] R. B. Ferroli, A. Mangoni, and S. Pacetti, Eur. Phys. J. C 80, 903 (2020). 